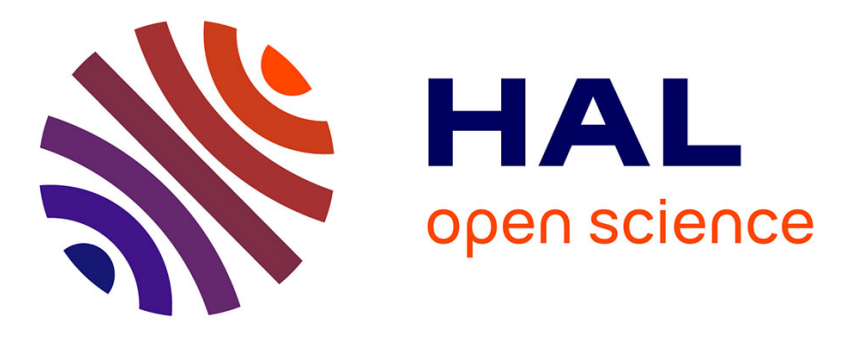

\title{
Embedding the Features of the Users' Physical Environments to Improve the Feeling of Presence in Collaborative Virtual Environments
}

Thierry Duval, Thi Thuong Huyen Nguyen, Cédric Fleury, Alain Chauffaut, Georges Dumont, Valérie Gouranton

\section{To cite this version:}

Thierry Duval, Thi Thuong Huyen Nguyen, Cédric Fleury, Alain Chauffaut, Georges Dumont, et al. Embedding the Features of the Users' Physical Environments to Improve the Feeling of Presence in Collaborative Virtual Environments. CogInfoCom 2012 (3rd IEEE International Conference on Cognitive Infocommunications), Dec 2012, Kosice, Slovakia. hal-00745598

\author{
HAL Id: hal-00745598 \\ https://hal.inria.fr/hal-00745598
}

Submitted on 1 May 2014

HAL is a multi-disciplinary open access archive for the deposit and dissemination of scientific research documents, whether they are published or not. The documents may come from teaching and research institutions in France or abroad, or from public or private research centers.
L'archive ouverte pluridisciplinaire HAL, est destinée au dépôt et à la diffusion de documents scientifiques de niveau recherche, publiés ou non, émanant des établissements d'enseignement et de recherche français ou étrangers, des laboratoires publics ou privés. 


\title{
Embedding the Features of the Users' Physical Environments to Improve the Feeling of Presence in Collaborative Virtual Environments
}

\author{
Thierry Duval \\ Université de Rennes I, UEB \\ IRISA, UMR CNRS 6074 \\ France \\ thierry.duval@irisa.fr
}

\author{
Huyen Nguyen \\ INRIA Rennes Bretagne-Atlantique \\ IRISA, UMR CNRS 6074 \\ France \\ thi-thuong-huyen.nguyen@inria.fr
}

\author{
Cédric Fleury \\ INSA de Rennes, UEB \\ IRISA, UMR CNRS 6074 \\ France \\ fleury@cs.unc.edu \\ (currently at University of North \\ Carolina at Chapel Hill, USA) \\ Valérie Gouranton \\ INSA de Rennes, UEB \\ IRISA, UMR CNRS 6074 \\ France \\ valerie.gouranton@irisa.fr
}

Georges Dumont

ENS Cachan antenne de Bretagne, UEB

IRISA, UMR CNRS 6074

France

georges.dumont@irisa.fr

\begin{abstract}
The feeling of presence is essential for efficient interaction within Virtual Environments (VE). When a user is fully immersed within a VE through a large display system such as a big $\mathrm{CAVE}^{\mathrm{TM}}$, his feeling of presence can be altered because of disturbing interactions with his physical environment, such as collision with hardware parts of the system or loss of tracking. This alteration can be avoided by taking into account the physical features of the user and to embed them in the VE. Moreover, the 3D representation of these physical features can also be useful for collaboration between distant users because they can make a user aware of the physical limitations of the other users they are collaborating with. In this paper we present how we use the Immersive Interactive Virtual Cabin (IIVC) model to obtain this virtual representation of the physical environment of the user and we illustrate how it can be used to guide efficiently a user for a navigation task in a VE.
\end{abstract}

\section{INTRODUCTION}

A 3D Virtual Environment (3D VE) is a virtual environment where 3D objects are displayed to a user. A user of such an environment is involved in a perception/action loop [1], and the success of his interactions contributes to his feeling of presence in the virtual environment [2]. Usually he can interact with this virtual environment through dedicated input devices. Chris Hand [3] proposes three categories of interactions: navigation (the interaction with the viewpoint of the user), manipulation of the virtual objects of the virtual environment (object selection, object manipulation), and application control (interaction with 3D widgets in order to change some parameters of the virtual environment). This is very similar to the four categories proposed by Bowman et al. [4] where interaction with the objects of the world is explicitly decomposed into selection and manipulation. Many efficient interaction techniques have been developed in this area in the past decade [5], and due to new 3D input devices and 3D displays becoming widely available for everyone, research in new 3D user interfaces is more relevant than ever [6]. When an interactive 3D Virtual Environment is deployed upon an immersive display system, such as a CAVE ${ }^{\mathrm{TM}}$ [7], or a HeadMounted Display (HMD), or a workbench, or simply a big screen, we talk about using Virtual Reality techniques in order to explore this 3D Virtual Environment and interact with it.

A 3D Collaborative Virtual Environment (3D CVE) is an interactive 3D virtual environment where several local or distant users can join to share a collaborative interaction experience. It can be considered as an infocommunication system, which can be used for communication between users and between users and (possibly remote) information. Object manipulation is a fundamental tasks of 3D interaction in Virtual Reality (VR), and collaborative manipulation of virtual objects by multiple users is a very promising area [8]. Collaborative manipulation of objects is indeed necessary in many different applications of VR such as virtual prototyping, training simulations or assembly and maintenance simulations [9]. In such virtual collaborative tasks, all the users should participate naturally and efficiently to the motion applied to the object manipulated in the VE [10], [11]. Another common use of 3D CVE is for virtual navigation: collaborative visits (museums, cultural heritage, architectural/urban project reviews) or collaborative games (cars races).

3D CVE intend to make the users not just remotely communicate, but rather really interact together by sharing interactions in the $3 \mathrm{D}$ virtual environment. These interactions can happen on distinct objects, or on different parts of a same 
object, or even on the same part (at the same time) of a shared virtual object [12].

In this paper section II presents a state of the art about providing awareness to users of VE. Section III recalls the IIVC concepts and how they can be used, while sections IV and V illustrates these concepts through an example of design and one example of implementation. Last, section VI concludes and gives future trends for this topic.

\section{AWARENESS IN ViRTUAL ENVIRONMENTS}

Embedding the user's motion workspace into the virtual environment offers the user an intuitive way to navigate by moving his own body. It also makes it possible to manage problems induced by the fact that the virtual world is often larger than this workspace. For example, the 3DM graphical modeler [13] enables a user to move on a "magic carpet" which represents the boundaries of the tracking area, he can perform real movements on the "magic carpet" to intuitively perform interactions. For long-distance navigation, he can also drive the "magic carpet" into the virtual world with a specific tool. For natural walking in virtual worlds with a restricted workspace, the "Magic Barrier Tape" [14] displays the boundaries of the physical workspace as a virtual barrier tape. It informs the user about the boundaries of his walking workspace defined by the tracking area or the display devices. Moreover, even if they do not display the user's motion workspace in the virtual environment, previous work about natural walking also has to consider these workspaces to prevent the user from colliding with the real environment or leaving the tracking area [15], [16].

Within collaborative virtual environments, comprehension problems can occur for users with different viewpoints on the virtual world [17]. Even if they can see each other user's avatar, its position, and its orientation in the virtual world as in CALVIN [18], users have difficulty in perceiving what the others see, and more generally what they are doing and what they can do. To overcome these perception problems, Fraser et al. [17] explicitly outline each user's view frustum using a wireframe model. By extension, the spatial model of interaction proposed by [19] can be seen as an interesting approach to describe users' multi-sensory perception. This spatial model defines sensory focus and nimbus for each user. The focus corresponds to the area in which a user has a sensory perception of the other users or of the virtual objects. The nimbus corresponds to the area in which the others have a sensory perception of this user. Moreover, users carry their focus and nimbus when they move in the virtual world.

To conclude, modeling users' physical environment improves user presence by matching the virtual world with the real world and by providing an environment safe from collisions or tracking problems. However, existing solutions do not deal with the representation of these devices in the virtual environment, and they can neither describe the spatial relationships between these physical devices, nor model the users' physical workspace associated to each device. Other solutions describe the organization of users' physical environment by a hierarchy of coordinate systems and introduce the notion of workspace, but they do not consider the physical workspaces of a user as explicit 3D volumes. So the notion of workspaces introduced by Mulder et al. [20] must be generalized to all the sensory workspaces and to various devices. This is why we need a generic model that enables VR developers to embed the users' physical environment into the VE when designing new applications, especially collaborative ones.

\section{THE IIVC MODEL}

We have proposed the Immersive Interactive Virtual Cabin (IIVC) [21] in order to cope with these problems. The IIVC is a generic solution that considers the users' physical environment during the VR software design, its deployment and its use. This solution provides a high-level model to describe, configure and modify the users' physical workspace organization whatever the immersive devices used.

We propose to model the users' physical environment as a structured hierarchy of virtual workspaces. Here we will focus mainly on the motion workspace (the area where a user can move his body), the visual workspace (what the user can see through and around a display device) and the interaction workspace (the area where a user can interact). We call stage the reference workspace of our hierarchy of workspaces that depicts the real-world spatial relationships between these workspaces.

The IIVC can be defined as an abstraction of the users' physical environment in the virtual world. It enables developers to implement their VR software without considering the physical devices used. For example, developers only have to manage position, orientation and scale of each user's IIVC when they develop navigation techniques. In a second step, each IIVC is configured with the features of each user's physical devices (size, shape, hierarchy of workspaces). The IIVC is based on three main components: the workspace, the stage, and the conveyor.

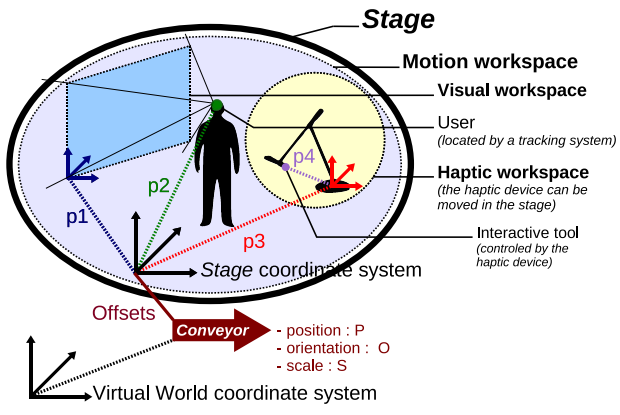

Fig. 1. The IIVC structure: the conveyor carries the stage with its workspaces in the virtual world.

The stage is a virtual description of the users' real environment. It usually matches the room where users interact, but it is also the virtual space containing the virtual representations of users' workspaces. These workspaces are defined by the features of the physical devices used. For example, motion workspace limits are often defined by the boundaries of the 
area in which users can move: position of the display devices (such as in CAVE ${ }^{\mathrm{TM}}$ [7] or a Reality Center) or limits of the tracking area. These workspaces are organized in a hierarchy of included 3D spaces into the stage. Each workspace has its own 3D shape and its own coordinate system to locate smaller workspaces or objects (real or virtual) that it contains. The stage uses its own coordinate system to locate directly or indirectly all the users' workspaces and all the objects of the IIVC. With this organization, the IIVC model is able to deal with physical reconfiguration such as modifications of workspace position and shape, additions of new screens or other devices, etc.

The conveyor is the integration frame of the stage into the virtual world. This conveyor is located in the virtual world coordinate system, so it has its own position, orientation and scale in this world. The stage is linked to the conveyor with position, orientation, and scale offsets (see Figure 1). The conveyor also defines the navigation technique, the travel direction, the rotation center, and the scale of the IIVC. So the stage, its workspaces and consequently the objects inside the workspaces are carried by the conveyor when it moves or changes its scale in the virtual world.

The conveyor is totally virtual, while the stage makes the link between the real world and the virtual world. With this splitting into two parts, we propose to define the limit of the stage as the last physical level which cannot move during the simulation. For example, in a CAVE ${ }^{\mathrm{TM}}$, the limit of the stage will be the cube defined by the screen's position.

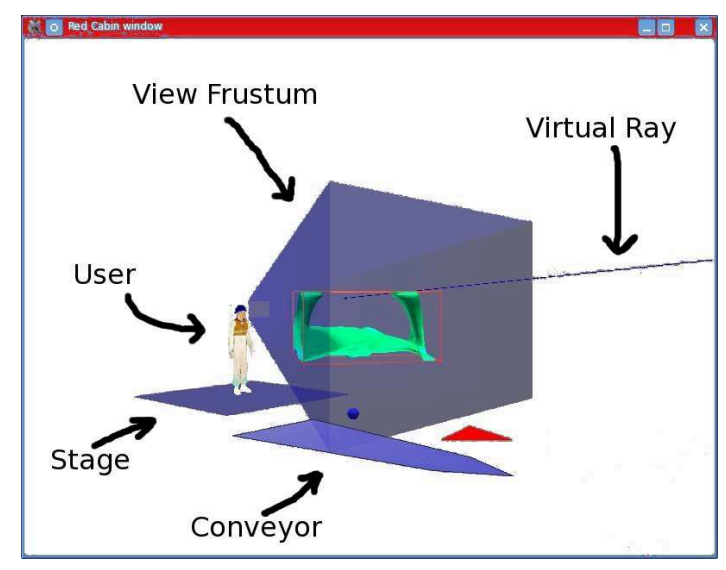

Fig. 2. One user within his IIVC viewed by another user: we can see him, his conveyor, his stage, his view frustum and his virtual ray.

Our model solves issues induced by collaborative sessions with remote users who interact from different physical environments: it enables users to perform a more effective collaboration by providing them a better understanding of the others' interaction capabilities, as it integrates users' physical workspaces and interaction tools in the virtual environment (see Figure 2).

\section{EXAMPle of Design of A 3D CVE}

For example, let's consider the design of a collaborative application where two distant users are going to share a co- manipulation of a virtual table through a 3-point manipulation technique. We will suppose that one user will use a big immersive system and that his head and his two hands will be tracked so that he will be able to use his two hands to drive 3D cursors (see Figure 3 (a)), while the other user will use a desktop system and a $2 \mathrm{D}$ input device (such as a $2 \mathrm{D}$ mouse) for driving a 3D ray and that his head will be tracked so that his point of view will change when he will move in front of his screen (see Figure 3 (b)).

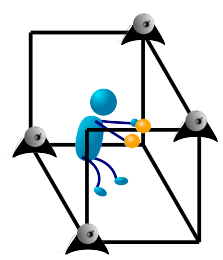

(a)

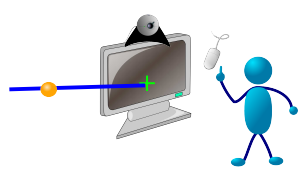

(b)
Fig. 3. (a) The first user in his big immersive system - (b) The second user with his desktop system

Indeed, the designer of such a distributed and multi-user application is facing several complex topics to meet its requirements, here we will focus only on interaction and collaboration: he must describe both the interactive content of the virtual universe and the collaborative interaction tools that will be used to drive this content, through dedicated new collaboration metaphors able to give to the users the best collaborative 3D interaction experience. Here, there will be a table, two 3D cursors, a 3D ray, an avatar of the position of the head of the first user, and an avatar of the position of the head of the second user. The 3D cursors and the 3D ray will be able to manipulate the table by using a 3-point manipulation technique [22].

Moreover, he also should be able to integrate a representation of the run-time hardware components in the virtual environment, to make the users aware of the limitations of these components. It leads to the creation of new virtual objects that must be added to the CVE: a representation of the bounds of the tracking systems of each user, a representation of the display surfaces of the first user, and a representation of the field of view of the second user, as illustrated in Figure 4.

Although it is important to work on abstract interaction and metaphors, indeed sometimes the hardware features of the real environment of the users must be embedded in the run-time VR application to take into account the physical environment of the users, such as the size and the resolution of the screens of a VR immersive system, as illustrated in detailed in Figure 4.

\section{IMPLEMENTATION EXAMPLE}

Collaboration can provide a powerful technique for exploration of large unknown virtual environments. It makes it possible to support the exploring user to deal with lack of spatial knowledge. Although Collaborative Virtual Environments (CVEs) have been developed to provide a framework 


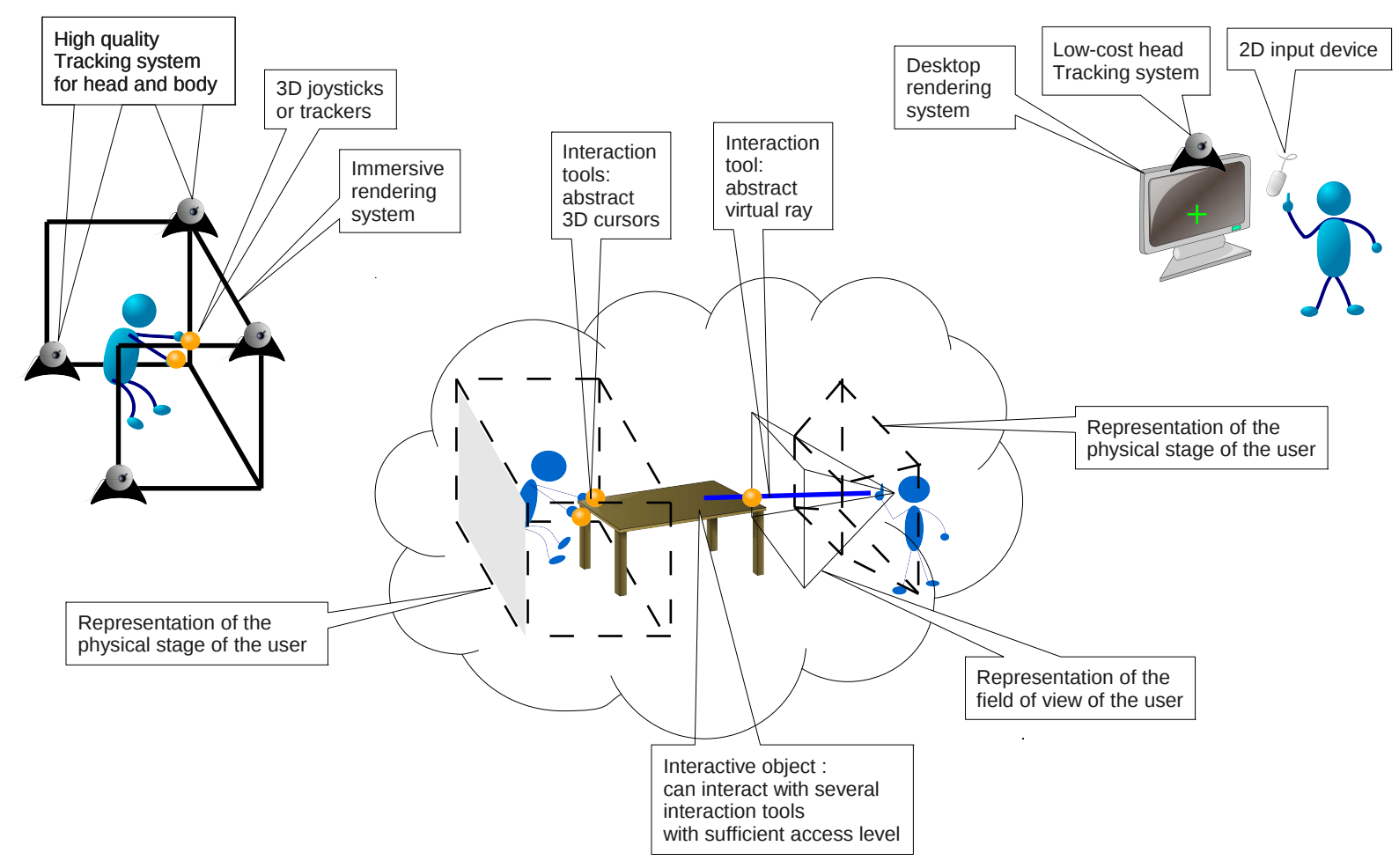

Fig. 4. Modeling collaborative interaction in a CVE.

of information sharing and communication [23], [24], [25], collaborative navigation task in such environments has not been largely explored and only limited attention has been devoted to evaluate its efficiency in navigation in VEs.

It is essential for navigation in a CVE to support communication between users because it is vital to understand what the others are referring to. So the communication technique for collaboration, especially for navigation in CVEs, should be simple, intuitive, efficient and non-verbal (because users do not always speak the same language). Based upon these points, our primary motive was to develop guiding techniques enabling helping users to guide an exploring user toward target places in complex large-scale CVEs. We share this objective with the organizers of the 3DUI Contest 2012 and its participants. As navigation aids, some techniques had been proposed such as "anchors" and a string of blue arrows that connects them or directional arrows [26], [27], point light sources [28] or light signal or beacons [29], [27], [30].

We have developed three guiding techniques in the form of navigation aids (arrows, light source and compass) that would enable one or several helping user(s) to guide an exploring user who is traveling in an unfamiliar 3D VE efficiently.

\section{A. Arrows}

The first guiding technique that we propose is based on directional arrows (see Figure 5) that are drawn in the view of the helping users to indicate the direction or the path that the exploring user has to follow. The helping users can draw as many directional arrows of different sizes as they want. However, so many directional arrows added within the environment or too big arrows may affect the immersion of the exploring user. As a result, the helping users have to determine when, how and where to put directional arrows to guide efficiently the exploring user. These arrows will disappear after a while. So the helping users are recommended to draw directional arrows within easy reach of the exploring user's visibility zone.

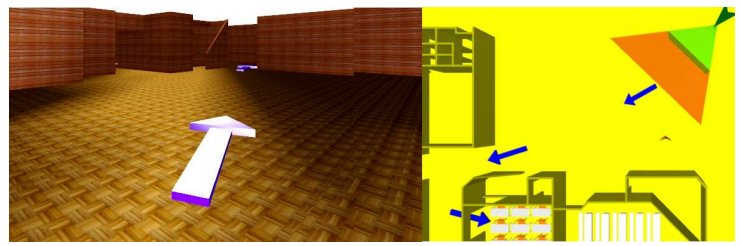

Fig. 5. Directional arrows in the exploring user's and the helping user's views.

\section{B. Light source}

The second guiding technique is based on a light source used as a beacon to light up a path to each target object (see Figure 6). The exploring user cannot see the light source itself but only its effect on objects within the environment. The light source is attached to a support (a 3D object) that can only be seen by a helping user. This helping user controls the light source by moving its support with a 3D cursor and shows up to the exploring user the path he must follow. It is important for the helping user to estimate if the exploring user can perceive the effect of the light on the environment. 


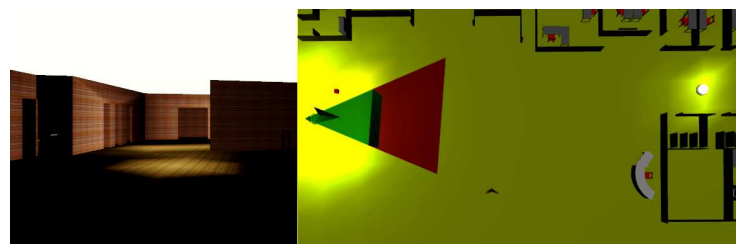

Fig. 6. Light source in the exploring user's and the helping user's views.

\section{Compass}

The third guiding technique is based on a compass attached to the position of the exploring user (with an offset), a typical tool to navigate in VEs (see Figure 7). The compass does not point directly to the target object location, but points to the location of another virtual object that plays the role of the "north" of this compass, and this object cannot be seen by the exploring user. A helping user can control this "north" by moving it with a 3D cursor, to show up to the exploring user the path he must follow. So by moving the "north" of the compass, a helping user can guide the exploring user to pass across hallways, rooms, doors, etc. before reaching the target position. It is thus a simple and powerful tool to guide the exploring user in any VE.

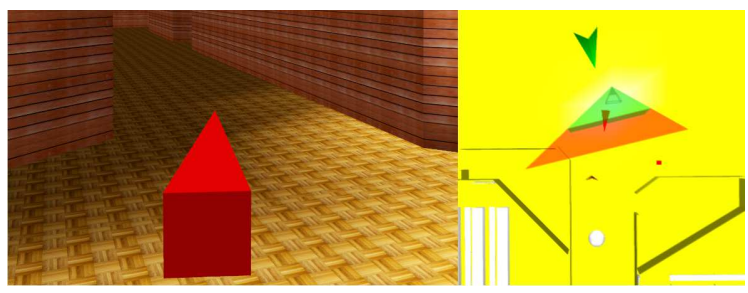

Fig. 7. Compass in the exploring user's and the helping user's views.

\section{The guiding viewpoints}

To be able to use these three guiding techniques in an efficient way, we built two principal kinds of views for our helping user: a bird's eye view (see the parts on the right of Figures 5, 6 and 7) and a first-person perspective by "looking over the exploring user's shoulder" (just like a camera attached to the shoulder of the exploring user) (see Figure 8). The bird's eye view can be considered as a 3D map or a WorldIn-Miniature [31]. To be used efficiently, these views must contain the 3D representation of some of the physical features of the stage of the exploring user, especially the position of his head which is used to build his virtual field of view. The representation of this field of view is dynamically reshaped while the user is moving inside his stage, and as this stage is attached to the virtual conveyor used for navigation, this stage moves with the conveyor, bringing with him the virtual field of view of the exploring user.

\section{CONCLUSION AND PERSPECTIVES}

In this paper we have explained why most Virtual Reality (VR) systems must consider the users' physical environment to immerse these users in a virtual world and to make them

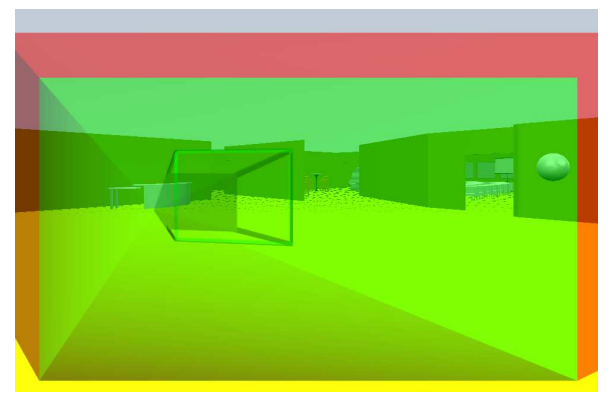

Fig. 8. A "looking over the exploring user's shoulder" view of the helping user.

aware of their interaction capabilities, which avoids disturbing interactions with their physical environment that would alter their feeling of presence. We propose to use the IIVC model that enables VR developers to embed the users' physical environment into the Virtual Environment (VE) when designing new applications, especially collaborative ones. Moreover, the 3D representation of these physical features can also be useful for collaboration between distant users because they can make a user aware of the physical limitations of the other users they are collaborating with. We have explained how to extract the physical features of the real environment of a user in order to embed them in the Virtual Environment and we have illustrated how these features can be exploited for a collaborative navigation task.

All these features are implemented in the Collaviz framework [32] dedicated to Collaborative Virtual Reality, which is available for all partners of the VISIONAIR ${ }^{1}$ project. Its implementation of the IIVC model makes it easy to install in any immersive system of this project, even if the partners prefer to use specific 3D API for rendering, as its architecture makes it possible to use several rendering engines [33]. Furthermore, its collaborative capabilities [34] make it possible to deploy collaborative applications between several VISIONAIR distant sites. Collaborative experiments have already been conducted between IRISA/INRIA Rennes (France) and University College of London (UCL) (England) [35].

Frameworks for collaborative virtual environments are now mature enough to allow researchers to focus on higher-level description of collaboration rather than on low-level system features. Establishing as automatically as possible a good matching between the virtual environment and the physical environment of the end-users is still a challenge and our IIVC concept is a first answer to this problem. However, describing all the components of an instance of the IIVC is still a consequent programming work, and a Model Driven Engineering (MDE) [36] approach would certainly be very interesting, with a Domain Description language (DSL) for describing the features of the physical environment and their matching with virtual objects of the virtual universe.

Last, we still have to improve the collaboration between distant users who are sharing a virtual environment, by propos-

\footnotetext{
${ }^{1}$ http://www.infra-visionair.eu/
} 
ing more efficient metaphors for 3D collaborative interactions. This topic is still very relevant: it was the main subject of the 3DUI 2012 contest. We participated to this contest by proposing some solutions based on the Collaviz framework in order to enhance collaboration between two users [27] and we will go on proposing new solutions dedicated to fully immersive collaboration.

\section{ACKNOWLEDGMENT}

We wish to thank Foundation Rennes 1 "Progress, Innovation, Entrepreneurship" and the European Project VISIONAIR (grant agreement 262044) for their support.

\section{REFERENCES}

[1] G. J. F. Smets, P. J. Stappers, and K. Overbeeke, "Designing in virtual reality: implementing perception-action coupling with affordances," in Proceedings of the conference on Virtual reality software and technology, ser. VRST '94. River Edge, NJ, USA: World Scientific Publishing Co., Inc., 1994, pp. 97-110.

[2] P. Zahorik and R. L. Jenison, "Presence as being-in-the-world," Presence: Teleoper. Virtual Environ., vol. 7, no. 1, pp. 78-89, Feb. 1998. [Online]. Available: http://dx.doi.org/10.1162/105474698565541

[3] C. Hand, "A Survey of 3D Interaction Techniques," Computer Graphics Forum, vol. 16, no. 5, pp. 269-281, 1997.

[4] D. A. Bowman, D. B. Johnson, and L. F. Hodges, "Testbed evaluation of virtual environment interaction techniques," in Proceedings of the ACM symposium on Virtual reality software and technology, ser. VRST '99. New York, NY, USA: ACM, 1999, pp. 26-33.

[5] D. A. Bowman, E. Kruijff, J. J. LaViola, and I. Poupyrev, 3D User Interfaces: Theory and Practice. Addison Wesley, 2004.

[6] D. Bowman, S. Coquillart, B. Froehlich, M. Hirose, Y. Kitamura, K. Kiyokawa, and W. Stuerzlinger, "3D User Interfaces: New Directions and Perspectives," Computer Graphics and Applications, IEEE, vol. 28 , no. 6, pp. 20-36, 2008.

[7] C. Cruz-Neira, D. J. Sandin, and T. A. DeFanti, "Surround-screen projection-based virtual reality: the design and implementation of the cave," in Proceedings of SIGGRAPH'93. New York, NY, USA: ACM, 1993, pp. 135-142.

[8] S. Benford, C. Greenhalgh, T. Rodden, and J. Pycock, "Collaborative Virtual Environments," Communication of the ACM, vol. 44, no. 7, pp. 79-85, 2001.

[9] R. A. Ruddle, J. C. D. Savage, and D. M. Jones, "Symmetric and Asymmetric Action Integration during Cooperative Object Manipulation in Virtual Environments," ACM Transactions on Computer-Human Interaction, vol. 9, no. 4, pp. 285-308, 2002.

[10] E. Frécon and A. A. Nöu, "Building distributed virtual environments to support collaborative work," in Proceedings of the ACM symposium on Virtual reality software and technology, ser. VRST '98. New York, NY, USA: ACM, 1998, pp. 105-113.

[11] V. Lalioti, C. Garcia, and F. Hasenbrink, "Virtual meeting in cyberstage," in Proceedings of the ACM symposium on Virtual reality software and technology, ser. VRST '98. New York, NY, USA: ACM, 1998, pp. 205-212.

[12] D. Margery, B. Arnaldi, and N. Plouzeau, "A General Framework for Cooperative Manipulation in Virtual Environments," in Virtual Environments'99, 1999, pp. 169-178.

[13] J. Butterworth, A. Davidson, S. Hench, and M. T. Olano, "3DM: A Three Dimensional Modeler using a Head-Mounted Display," in Proc. of the Symp. on Interactive 3D graphics, 1992, pp. 135-138.

[14] G. Cirio, M. Marchal, T. Regia-Corte, and A. Lécuyer, "The Magic Barrier Tape: A Novel Metaphor for Infinite Navigation in Virtual Worlds with a Restricted Walking Workspace," in Proc. of the 16th Symp. on Virtual Reality Software and Technology, 2009, pp. 155-162.

[15] S. Razzaque, "Redirected Walking," Ph.D. dissertation, University of North Carolina at Chapel Hill, 2005.

[16] B. Williams, G. Narasimham, B. Rump, T. P. McNamara, T. H. Carr, J. Rieser, and B. Bodenheimer, "Exploring Large Virtual Environments with an HMD When Physical Space is Limited," in Proc. of the 4th Symp. on Applied perception in graphics and visualization, 2007, pp. $41-48$.
[17] M. Fraser, S. Benford, J. Hindmarsh, and C. Heathq, "Supporting Awareness and Interaction through Collaborative Virtual Interfaces," in Proc. of the 12th Symp. on User Interface Software and Technology, 1999 , pp. 27-36.

[18] J. Leigh, A. Johnson, C. Vasilakis, and T. DeFanti, "Multi-perspective Collaborative Design in Persistent Networked Virtual Environments," in Proceedings of VRAIS'96, 1996, pp. 253-260.

[19] S. Benford, J. Bowers, L. E. Fahlén, and C. Greenhalgh, "Managing Mutual Awareness in Collaborative Virtual Environments," in Proc. of the Symp. on Virtual Reality Software and Technology, 1994, pp. 223236.

[20] J. D. Mulder and B. R. Boschker, "A Modular System for Collaborative Desktop VR/AR with a Shared Workspace," Proc. of the IEEE Virtual Reality Conference, vol. 0, p. 75, 2004.

[21] C. Fleury, A. Chauffaut, T. Duval, V. Gouranton, and B. Arnaldi, "A Generic Model for Embedding Users' Physical Workspaces into MultiScale Collaborative Virtual Environments," in Proc. of ICAT, 2011, pp. $1-8$.

[22] L. Aguerreche, T. Duval, and A. Lécuyer, "3-Hand Manipulation of Virtual Objects," in Proc. of JVRC 2009 (Joint Virtual Reality Conference of EGVE - ICAT - EuroVR), 2009, pp. 153-156.

[23] M. Macedonia, M. J. Zyda, D. Pratt, P. Barham, and S. Zeswitz, "Npsnet: A network software architecture for large scale virtual environments," Presence, vol. 3, no. 4, pp. 265-287, 1994.

[24] C. Dumas, S. Degrande, G. Saugis, C. Chaillou, M.-L. Viaud, and P. Plenacoste, "Spin: a 3d interface for cooperative work," Virtual Reality Society Journal, 1999.

[25] E. F. Churchill, D. N. Snowdon, and A. J. Munro, Collaborative Virtual Environments: Digital Places and Spaces for Interaction. London, United Kingdom: Springer Verlag London Ltd, 2001.

[26] F. Bacim, E. D. Ragan, C. Stinson, S. Scerbo, and D. A. Bowman, "Collaborative navigation in virtual search and rescue," in Proceedings of IEEE Symposium on 3D User Interfaces. IEEE Computer Society, March 2012, pp. 187-188.

[27] T. T. H. Nguyen, C. Fleury, and T. Duval, "Collaborative Exploration in a Multi-Scale Shared Virtual Environment," in Proceedings of IEEE Symposium on 3D User Interfaces. IEEE Computer Society, March 2012, pp. 181-182.

[28] M. Cabral, G. Roque, D. Santos, L. Paulucci, and M. Zuffo, "Point and go: Exploring 3d virtual environments," in Proceedings of IEEE Symposium on $3 D$ User Interfaces. IEEE Computer Society, March 2012, pp. 183-184.

[29] S. Notelaers, T. D. Weyer, P. Goorts, and S. Maesen, "Heatmeup: a 3dui serious game to explore collaborative wayfinding," in Proceedings of IEEE Symposium on 3D User Interfaces. IEEE Computer Society, March 2012, pp. 177-178.

[30] J. Wang, R. Budhiraja, O. Leach, R. Clifford, and D. Matsua, "Escape from meadwyn 4: A cross-platform environment for collaborative navigation tasks," in Proceedings of IEEE Symposium on 3D User Interfaces. IEEE Computer Society, March 2012, pp. 179-180.

[31] R. Stoakley, M. J. Conway, and R. Pausch, "Virtual reality on a wim: Interactive worlds in miniature," in Proceedings of the SIGCHI conference on Human Factors in Computing Systems. ACM Press/Addison-Wesley Publishing Co. New York, 1995, pp. 265-272.

[32] F. Dupont, T. Duval, C. Fleury, J. Forest, V. Gouranton, P. Lando, T. Laurent, G. Lavoué, and A. Schmutz, "Collaborative Scientific Visualization: The COLLAVIZ Framework," in JVRC Demos, 2010.

[33] T. Duval and C. Fleury, "Collaboration between networked heterogeneous $3 \mathrm{~d}$ viewers through a pac-c3d modeling of the shared virtual environment," in Proceedings of International Conference on Artificial Reality and Telexistence (ICAT). International Conference on Artificial Reality and Telexistence, 2011.

[34] C. Fleury, T. Duval, V. Gouranton, and B. Arnaldi, "A New Adaptive Data Distribution Model for Consistency Maintenance in Collaborative Virtual Environments," in Proc. of JVRC, 2010, pp. 29-36.

[35] C. Fleury, T. Duval, V. Gouranton, and A. Steed, "Evaluation of Remote Collaborative Manipulation for Scientific Data Analysis," in VRST 2012 - 18th Symposium on Virtual Reality Software and Technology. Toronto, Canada: ACM, Dec. 2012.

[36] J.-M. Jézéquel, O. Barais, and F. Fleurey, "Model Driven Language Engineering with Kermeta," in 3rd Summer School on Generative and Transformational Techniques in Software Engineering. LNCS 6491, Springer, 2010. 